\title{
Disentangling substance use and related problems: urgency predicts substance- related problems beyond the degree of use
}

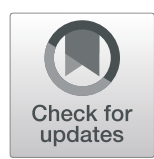

\author{
Malin K. Hildebrandt ${ }^{*}$ Raoul Dieterich and Tanja Endrass
}

\begin{abstract}
Background: Substance use disorders are reliably associated with high impulsivity and sensation seeking. Importantly, both precede problematic substance use, implicating them as risk factors. Individuals with substance use disorders show variable degrees of substance use (combined quantity and frequency) and substance-related problems and differ in both aspects from healthy controls. Dimensional research has indicated differential associations of impulsivity-related traits as well as sensation seeking with the degree of substance use and substance-related problems. The current study aimed to clarify whether impulsivity-related traits and sensation seeking predict substance-related problems above and beyond the degree of substance use and are thus specifically linked to problems, the dimension that characterizes substance use disorders.
\end{abstract}

Method: We assessed impulsivity-related traits and sensation seeking using self-report, as well as delay discounting, a behavioral indicator of impulsivity, in a sample of 258 substance-using adults.

Results: Sensation seeking and impulsivity-related traits significantly predicted the degree of substance use, with sensation seeking explaining the largest portion of variance. In contrast, self-reported impulsivity, in particular when experiencing negative emotions (urgency), but not sensation seeking or delay discounting, predicted substancerelated problems when controlling for the degree of substance use.

Conclusions: This suggests that urgency, but not sensation seeking, may be specifically linked to substance-related problems and thus especially relevant for substance use disorders. Taken together, this study underlines the necessity to assess and control for the degree of substance use in risk factor research concerning substance-related problems. Thus, it may inform future research improving targeted prevention and therapy.

Keywords: Substance use, Impulsivity, Sensation seeking, Dimensional, Urgency

\section{Background}

Substance use disorders (SUDs) are characterized not only by substance use itself but by problems related to substance use [1], such as the inability to control substance intake, or the failure to comply with social responsibilities. Not all frequent substance users develop

\footnotetext{
* Correspondence: malin.hildebrandt@tu-dresden.de Institute of Clinical Psychology and Psychotherapy, Chair of Addiction Research, Faculty of Psychology, Technische Universität Dresden, Chemnitzer Str. 46a, 01187 Dresden, Germany
}

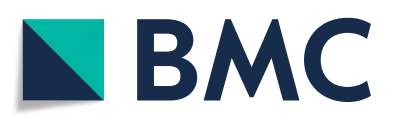

(c) The Author(s). 2021 Open Access This article is licensed under a Creative Commons Attribution 4.0 International License, which permits use, sharing, adaptation, distribution and reproduction in any medium or format, as long as you give appropriate credit to the original author(s) and the source, provide a link to the Creative Commons licence, and indicate if changes were made. The images or other third party material in this article are included in the article's Creative Commons licence, unless indicated otherwise in a credit line to the material. If material is not included in the article's Creative Commons licence and your intended use is not permitted by statutory regulation or exceeds the permitted use, you will need to obtain permission directly from the copyright holder. To view a copy of this licence, visit http://creativecommons.org/licenses/by/4.0/. The Creative Commons Public Domain Dedication waiver (http://creativecommons.org/publicdomain/zero/1.0/) applies to the data made available in this article, unless otherwise stated in a credit line to the data. geted prevention and treatment to identify factors that specifically influence the development of problems. Most of the literature on SUDs relies on comparisons of individuals diagnosed with SUDs and healthy controls in order to do so. Here, the patients differ from healthy controls in both the degree of substance use (quantity and frequency) and substance-related problems. This hinders evaluating whether the examined potential risk factors are truly associated with the development of 
substance-related problems, the dimension that is critical for the diagnosis of SUDs. The specific associations of potential risk factors with the degree of substance use and substance-related problems remain unclear. Here, we are proposing a dimensional approach examining the associations of potential risk factors with substancerelated problems while statistically controlling for the degree of substance use. Consequently, we assessed both variables separately and dimensionally in a population of substance users of varying degrees of substance use. Using this approach, we aimed to take a first step in reevaluating the role of impulsivity-related traits as risk factors for SUDs. High levels of impulsivity-related traits are robustly found in individuals with SUDs [2-5]. So far, it has never been tested whether this is driven by the association of these factors with the degree of substance use or whether they are, beyond that, incrementally relevant for substance-related problems across different substances. Particularly, controlling for the degree of use has been neglected in previous work.

Overall, impulsivity is robustly associated with SUDs (for a review see e.g. [6]). However, in the relevant literature, the conceptualization of impulsivity is not always coherent and hence impulsivity may be best described as an umbrella term subsuming several related traits. In an attempt to solve this empirically, Whiteside and Lynam [7] were the first to conduct a factor analysis of various questionnaires assessing impulsivity. This analysis and similar factor analyses repeatedly suggested that impulsivity may be divided into four distinct, yet related traits which constitute the UPPS model of impulsivity: Urgency, (lack of) Premeditation, (lack of) Perseverance and Sensation seeking $[7,8]$. The first three traits conform with a narrow definition of impulsivity, understood as the propensity to act quickly while disregarding longterm negative consequences [9] and we will therefore subsume them as impulsivity-related traits. More specifically, urgency describes the tendency to act rashly when experiencing negative emotions, (lack of) premeditation describes the tendency to act without thinking, and (lack of) perseverance describes the tendency not to finish tasks [10]. Sensation seeking does not conform with this narrow definition of impulsivity and has recently been pinpointed as a separate construct [11] that correlates only weakly with impulsivity-related traits (between $\mathrm{r}=$ 0.00 and $r=0.18[7,12] ;)$, suggesting that the tendency to seek excitement is to a large extent independent of the tendency to act rashly. The UPPS model is useful for a comprehensive assessment of impulsivity as it builds on several conceptualizations of impulsivity from the literature and other conceptualizations can be allocated to the UPPS traits (see e.g. [13]). For example, regarding the widely used Barratt Impulsiveness Scale [14], the "lack of planning" and "attentional impulsivity" subscales can be allocated to the traits (lack of) premeditation and urgency, respectively.

Longitudinal studies suggest that sensation seeking prospectively predicts the risk to develop SUDs (e.g .[4]). Further studies indicate impulsivity-related traits as risk factor for SUDs by showing that impulsivity-related traits prospectively predict the degree of substance use as well as related problems with similar $[15,16]$ or higher [17] associations of impulsivity-related traits with problems than with the degree of substance use. Notably, substance use and problems do not prospectively predict impulsivity-related traits [18]. One study provides first evidence that a composite score that included impulsivity-related measures, sensation seeking and emotional control prospectively predicts substancerelated problems even when controlling for substance use (with a medium effect size [16];).

Research further suggests that impulsivity-related traits as well as sensation seeking are differentially related to the degree of substance use and problems. Sensation seeking and premeditation are associated with the degree of substance use $[8,19,20]$. A meta-analysis that assigned various impulsivity measures to the traits of the UPPS model found that all traits were similarly related to the degree of use, while urgency was the trait most strongly related to problems [13]. The trait of urgency, specifically when experiencing negative emotions, as it was conceptualized in the UPPS model, has also been linked to other types of problem behavior (e.g. bulimic symptoms, problematic gambling, self-injury and sexual risk taking [21-23];). Moreover, although the evidence is so far restricted to alcohol use, urgency and perseverance were shown to be associated with problems, even when controlling for the degree of substance use [20]. Importantly, the association of sensation seeking with problems disappeared when the degree of substance use was statistically controlled for. These findings underline that the approach we propose here is suitable for identifying factors specifically associated with substancerelated problems.

One widely studied behavioral indicator of impulsivity is delay discounting, the subjective devaluation of rewards based on how far in the future they become available [24]. This index of the preference for smaller sooner over larger later rewards is of high conceptual relevance to SUD research, as it closely resembles behavior observed in individuals with SUDs, i.e., choosing immediate drug effects over long-term goals (e.g., taking care of family or school/job etc.). Empirically, higher delay discounting in individuals with SUDs [25] and associations of delay discounting with substance use frequency [26] and SUD severity (indicating substancerelated problems) are consistently observed [27-29]. Delay discounting correlates with premeditation [19, 
30-32], but also urgency [30] and sensation seeking [32], but a meta-analysis suggests that these associations are overall rather weak [32].

The goal of this study was to extend the finding that impulsivity-related traits, but not sensation seeking, predict alcohol-related problems above and beyond the degree of substance use [20]. We investigated this relationship in an online survey in a sample of undiagnosed (poly-)substance users with widely varying degrees of substance use. Thereby, we aimed to examine if impulsivity-related traits, but not sensation seeking, may be specifically linked to substance-related problems (and thus truly be relevant for SUDs) across different substances. Furthermore, we assessed delay discounting to examine whether selfreported and behavioral impulsivity-related traits distinctly relate to substance use variables. We expected that impulsivity-related traits (self-report and delay discounting) as well as sensation seeking would predict the degree of substance use, while only impulsivity-related traits (more specifically urgency, perseverance and delay discounting), but not sensation seeking, would predict substance-related problems when controlling for the degree of substance use.

\section{Method}

\section{Participants}

Participants were recruited mainly through advertisements in clubs, webpages associated with the techno music scene, and counseling centers as well as through postings and flyers. Inclusion criteria were assessed as self-report questions in the online survey and were: (1) current use of at least one substance at least once a month, (2) age between 18 and 40 years, (3) native German speakers, (4) no reported use of any substance (except for nicotine) on the day of the survey. We excluded these participants to avoid effects of acute intoxication on questionnaire responses. The final sample, after excluding those survey participants who did not fulfill the inclusion criteria $(N=114)$ consisted of 258 participants.

Participants reported a wide range of substance use frequency, quantity and related problems. As this was an online survey, we did not confirm SUD criteria by a diagnostic interview. Hence, the current sample is a non-clinical and undiagnosed sample, but likely includes individuals at risk for SUD diagnosis as indicated by the range of problems participants reported (see Fig. 1). Moreover, 54.7\% reported frequent use (at least three to four times a week) of at least one substance. See Fig. 1 and Table 1 for sociodemographic and substance userelated characteristics of participants. Further details are available in the supplementary material.

Participants gave informed consent prior to participation and could earn course credit (six participants) or take part in a lottery to win one of three $10 €$ vouchers. The study protocol was approved by the local ethics committee (EK 146042019) and followed the guidelines stated by the Declaration of Helsinki.

\section{Procedure}

Participants completed an online-survey (approx. 20 min) comprising sociodemographic information, inclusion criteria, and the measures described below.

\section{Material}

\section{Substance use questionnaire}

This questionnaire measured the frequency and quantity of substance use for the following substance classes from the DSM-5: alcohol, tobacco, cannabis, stimulants, opioids, hallucinogenic substances, misused medication, inhalants, other substances. Participants were first asked whether they had ever used any of the substances from the respective substance class. If they answered 'yes', participants indicated, among other variables not relevant here, their average frequency of use within the past three months on a 6-point Likert scale ranging from 1 to 6 (almost daily, 3-4 times a week, 1-2 times a week, 1-2 times a month, less than once a month, never) and the average subjective quantity of use on a standard occasion within the past three months on a 5-point Likert scale ranging from 1 (very little) to 5 (very much). We computed individual degree of substance use scores for each substance class (product of quantity and frequency values, possible maximum: 30) as an intermediate step and summed these scores to compute the total degree of substance use score which was used in the analyses (possible maximum: 270).

\section{Substance-related problems questionnaire}

This questionnaire measured substance-related problems operationalized by the number of DSM-5 SUD symptoms from the A criterion participants reported to have experienced within the past 12 months (range 0-11). Questions were based on the German version of the Structured Clinical Interview for DSM-5 Disorders Clinician Version [33] and adapted to the online application. Participants were instructed to consider all substances when judging whether they had experienced each symptom. Thus, this index does not equal a diagnosis of a specific substance use disorder, but rather an index of problem severity across substances as experienced symptoms may be attributable to different substances.

\section{UPPS impulsive behavior scale}

This 45 -item self-report questionnaire assesses four subscales representing facets of impulsivity as identified by Whiteside and Lynam [10]: [1] negative urgency (e.g., "In the heat of an argument, I will often say things that I later regret."; possible range: 12-48), [2] (lack of) 


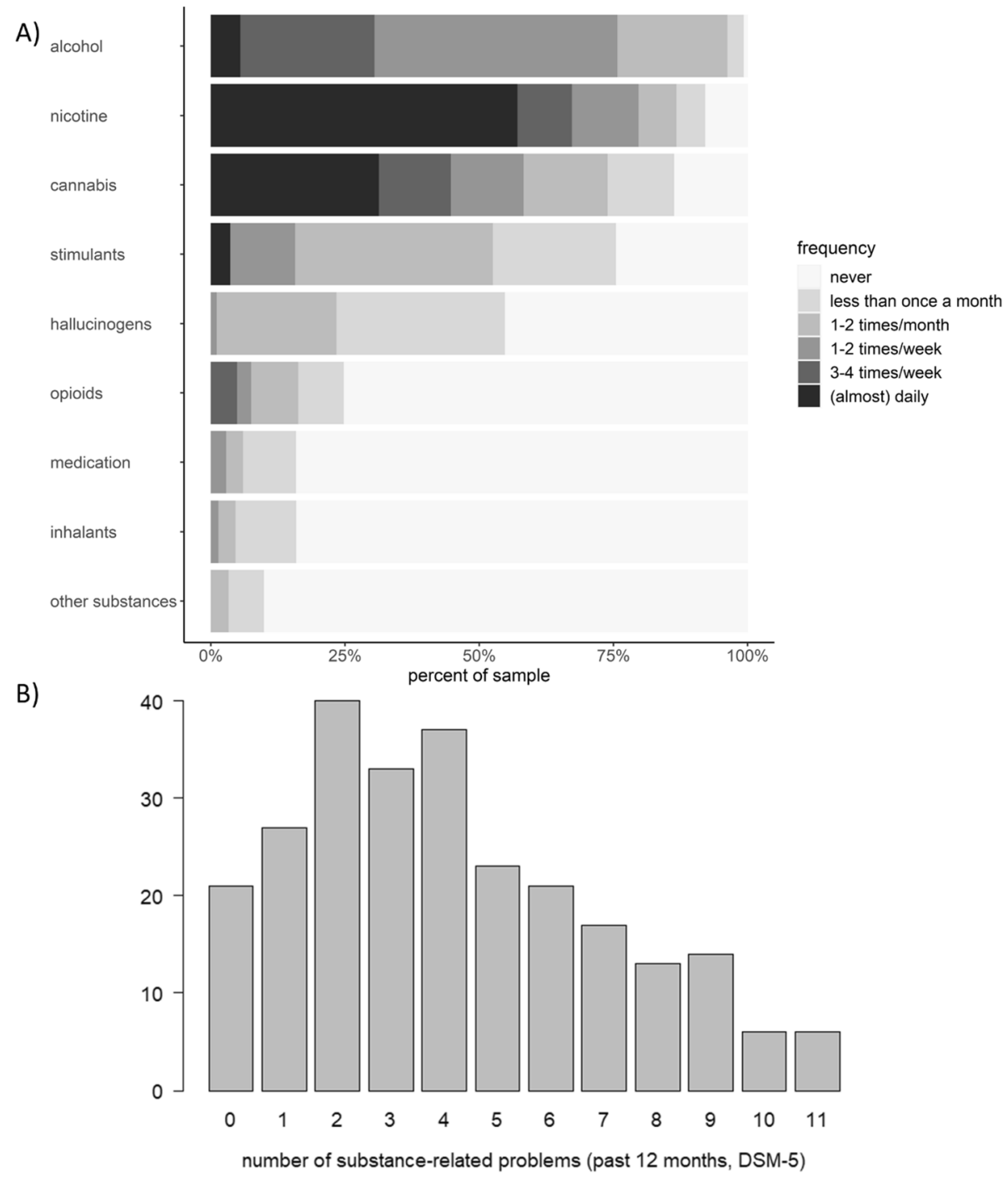

Fig. 1 Descriptive information on substance use and substance-related problems. Note. a. Distribution of substance use frequency in percent separately for each substance class within the current sample. b. Histogram of substance-related problems: the $y$-axis depicts the number of participants reporting the respective cumulative number of self-reported substance-related problems (DSM- 5 criteria, irrespective of specific substance). Note that substance-related problems were assessed across substances and thus $\geq 2$ problems do not entail an SUD diagnosis

premeditation (e.g., "I usually make up my mind through careful reasoning."; possible range: 11-44), [3] (lack of) perseverance (e.g., "Once I start a project, I almost always finish it."; possible range: 10-40), and [4] sensation seeking (e.g., "I welcome new and exciting experiences and sensations, even if they are a little frightening and unconventional."; possible range: 11-44). Items are rated on a 4-point Likert scale ranging from 1 (strongly agree) to 4 (strongly disagree). If necessary, items are reverse coded so that higher values on a subscale always indicate higher impulsivity/sensation seeking. The subscales of the German version [34] have good internal consistency (cronbachs $\alpha=.81-.85$ ) and external validity [35].

\section{Monetary choice questionnaire (MCQ)}

The MCQ (German version [36];) assesses delay discounting. It comprises 27 binary choices between varying amounts of money (8-66 Euros) available either now or with a delay (7-238 days). Values and delays of the options are predefined to cover a high range of discounting rates for low, medium, and high values of delayed rewards, respectively. The discounting rate $k$ can be modeled assuming a hyperbolic function [37].

$$
V=\frac{A}{1+k D}
$$


Table 1 Sociodemographic and substance use-related characteristics of participants

\begin{tabular}{|c|c|c|c|c|}
\hline \multicolumn{3}{|l|}{ Characteristic } & $N$ & $\%$ \\
\hline \multicolumn{5}{|l|}{ Gender } \\
\hline \multicolumn{2}{|l|}{ female } & & 148 & 57.4 \\
\hline \multicolumn{2}{|l|}{ male } & & 109 & 42.2 \\
\hline \multicolumn{2}{|l|}{ not specified } & & 1 & 0.4 \\
\hline \multicolumn{5}{|l|}{ Secondary school degree } \\
\hline \multicolumn{2}{|l|}{ upper } & & 226 & 87.6 \\
\hline \multicolumn{2}{|l|}{ intermediate } & & 28 & 10.9 \\
\hline \multicolumn{2}{|l|}{ lower } & & 2 & 0.8 \\
\hline \multicolumn{5}{|l|}{ Current use of } \\
\hline \multicolumn{2}{|l|}{ alcohol } & & 236 & 91.5 \\
\hline \multicolumn{2}{|l|}{ nicotine } & & 159 & 61.6 \\
\hline \multicolumn{2}{|l|}{ cannabis } & & 117 & 45.3 \\
\hline \multicolumn{2}{|l|}{ stimulants } & & 79 & 30.6 \\
\hline \multicolumn{2}{|l|}{ hallucinogenic substances } & & 29 & 11.2 \\
\hline \multicolumn{2}{|l|}{ opioids } & & 14 & 5.4 \\
\hline \multicolumn{2}{|l|}{ misused medication } & & 5 & 1.9 \\
\hline \multicolumn{2}{|l|}{ inhalants } & & 4 & 1.6 \\
\hline \multirow[t]{2}{*}{ other substances } & & & 3 & 1.2 \\
\hline & M & MD & SD & range \\
\hline age & 26.1 & 25.0 & 5.0 & $18-40$ \\
\hline currently used substance classes & 2.5 & 2.0 & 1.3 & $1-6$ \\
\hline substance classes used lifetime & 4.7 & 5.0 & 2.0 & $1-9$ \\
\hline total degree of substance use & 35.9 & 36.5 & 19.4 & $3-89$ \\
\hline substance-related problems & 4.2 & 4 & 2.9 & $0-11$ \\
\hline
\end{tabular}

Note. $M, M D$, and $S D$ represent mean, median, and standard deviation, respectively. Note that substance-related problems were assessed across substances and thus $\geq 2$ problems do not entail an SUD diagnosis

where $V$ is the subjective value of a delayed reward with the amount $A$ after the delay $D$.

The parameter $k$ is estimated based on the proportion of choices that are consistent with every possible value of $k$ (for a thorouh description please refer to [38]). As $k$ is dependent on value [39], $k$ is estimated for small, medium and large delayed rewards, respectively, and the geometric mean of these values constitutes an individual's $k$-value.

\section{Data analysis}

All analyses were conducted in $\mathrm{R}$ [40]. We logtransformed the $k$-parameter due to skewedness. Pearson's correlations were computed to examine bivariate associations. Two-tailed tests of the difference between two dependent correlations with one variable in common were conducted to test whether correlations differed significantly. Subsequently, we computed partial correlations of all predictor variables with substance-related problems controlled for the degree of substance use to explore their respective contribution outside the context of other predictor variables. Last, we conducted two hierarchical linear regressions for the degree of substance use and substancerelated problems to identify the unique variance explained by sensation seeking, impulsivity-related traits, and $\log (k)$. For both regression models, we entered gender as a covariate in the first step, because it was significantly related to both outcome variables, while age was not (ps $>.05$ ). We excluded the group "gender not specified" from these regression analyses $(n=1)$. For the model predicting the degree of substance use, we added the UPPS subscales in the second and $\log (k)$ in the third step. For the model predicting substance-related problems, we added the degree of substance use as a covariate in the second step, the UPPS subscales in the third step and $\log (k)$ in the fourth step. To test on an exploratory basis whether a regressor explained more variance in the criterion variable than another regressor, we computed finite-sample F-statistics using the "linearHypothesis" function from the car package [41]. We applied a significance level of $\alpha=.05$ for all analyses.

\section{Results}

\section{Bivariate correlations}

Discounting rates ranged from 0.000158 to 0.24853 , with some choosing exclusively the smaller sooner or the larger later option, and were not significantly associated with any of the UPPS subscales (all $p \mathrm{~s}>.05$ ), except for a small positive correlation with urgency (see Table 2 for an overview of correlations). Delay discounting and (lack of) premeditation were significantly, but weakly, correlated with both the degree of substance use and problems. (Lack of) perseverance displayed stronger correlations (small to moderate) with both substance use variables. Sensation seeking correlated significantly stronger with the degree of substance use than with problems, $r=.38$ vs. $r=.23$, $t(256)=2.72, p=.01$. In turn, urgency correlated significantly stronger with substance-related problems than with the degree of substance use, $\mathrm{r}=.33$, vs. $\mathrm{r}=.18, t(256)=$ $2.67, p=.01$. The degree of substance use and substancerelated problems were strongly correlated.

\section{Partial correlations}

When controlling for the degree of substance use, urgency $(r(256)=.28, p<.001)$ and perseverance $(r(256)=$ $.20, p=.001)$ significantly correlated with substancerelated problems, while premeditation, sensation seeking and $\log (k)$ did not (all $p \mathrm{~s}>.05$ ).

\section{Relative contributions of UPPS subscales and delay discounting to substance use outcomes The degree of substance use}

In the first model, we regressed the degree of substance use on the UPPS subscales and $\log (k)$ while controlling 
Table 2 Means, Standard Deviations, and Correlations with Confidence Intervals for UPPS subscales, Delay Discounting (log(k)), the Degree of Substance Use and Substance-Related Problems

\begin{tabular}{|c|c|c|c|c|c|c|c|c|c|}
\hline & Variable & $M$ & $S D$ & 1 & 2 & 3 & 4 & 5 & 6 \\
\hline \multicolumn{10}{|c|}{ UPPS } \\
\hline 1. & Urgency & 26.77 & 5.96 & & & & & & \\
\hline \multirow[t]{2}{*}{2.} & Premeditation & 23.59 & 4.50 & $.32^{* *}$ & & & & & \\
\hline & & & & {$[.20, .42]$} & & & & & \\
\hline \multirow[t]{2}{*}{3.} & Persistence & 20.40 & 4.41 & $.40^{* *}$ & $.26^{* *}$ & & & & \\
\hline & & & & {$[.29, .50]$} & {$[.14, .37]$} & & & & \\
\hline \multirow[t]{2}{*}{4.} & Sensation Seeking & 32.00 & 7.26 & -.00 & $.32^{* *}$ & .01 & & & \\
\hline & & & & {$[-.12, .12]$} & {$[.21, .43]$} & {$[-.12, .13]$} & & & \\
\hline \multicolumn{10}{|c|}{ MCQ } \\
\hline \multirow[t]{2}{*}{5.} & $\log (k)$ & -2.28 & 0.75 & $.14^{*}$ & -.00 & .04 & -.01 & & \\
\hline & & & & {$[.01, .25]$} & {$[-.12, .12]$} & {$[-.08, .16]$} & {$[-.14, .11]$} & & \\
\hline \multicolumn{10}{|c|}{ Substance use variables } \\
\hline \multirow[t]{2}{*}{6.} & Degree of use & 35.89 & 19.36 & $.18^{* *}$ & $.16^{*}$ & $.22^{* *}$ & $.38^{* *}$ & $.18^{* *}$ & \\
\hline & & & & {$[.05, .29]$} & {$[.04, .28]$} & {$[.10, .34]$} & {$[.27, .48]$} & {$[.06, .30]$} & \\
\hline \multirow[t]{2}{*}{7.} & Problems & 4.15 & 2.85 & $.33^{* *}$ & $.13^{*}$ & $.29^{* *}$ & $.23^{* *}$ & $.15^{*}$ & $.55^{* *}$ \\
\hline & & & & {$[.22, .43]$} & {$[.01, .25]$} & {$[.17, .39]$} & {$[.11, .35]$} & {$[.03, .27]$} & {$[.46, .63]$} \\
\hline
\end{tabular}

Note. $M$ and SD represent mean and standard deviation, respectively. Values in square brackets indicate the $95 \%$ confidence interval. UPPS Urgency Premeditation Perseverance Sensation Seeking Impulsive Behavior Scale, MCQ Monetary Choice Questionnaire, $k=$ delay discounting. ${ }^{*} p<.05$. ${ }^{* *} p<.01$

for gender (see Table 3). The hierarchical multiple regression revealed that all UPPS subscales (except for premeditation) as well as $\log (k)$ explained significant unique variance in the degree of substance use, such that higher levels of urgency, perseverance, sensation seeking and delay discounting were associated with more substance use. In the complete model, sensation seeking had a significantly stronger effect on the degree of substance use than all other impulsivity-related predictors respectively, all $F \mathrm{~s}>4.13$, all $p \mathrm{~s}<.05$. The complete model accounted for $25 \%$ of the variance in the degree of substance use.

\section{Substance-related problems}

In the second model, we regressed substance-related problems on the UPPS subscales and $\log (k)$ while controlling for age and the degree of substance use (see Table 3). The hierarchical multiple regression revealed that the UPPS urgency subscale explained significant unique variance in substance-related problems, such that higher levels of urgency were associated with more problems above and beyond the degree of substance use, which also predicted substance-related problems. None of the other predictors were significantly associated with substance-related problems. In the complete model, urgency had a significantly stronger effect on substancerelated problems than premeditation and $\log (\mathrm{k})$ (all $F \mathrm{~s}>$ 5.57 , all $p \mathrm{~s}>.05$ ) and sensation seeking, but this difference was only a marginally significant $(F=2.96, p=.09)$.
The complete model accounted for $39 \%$ of the variance in substance-related problems.

\section{Discussion}

This study examined the differential associations of impulsivity-related traits, sensation seeking, and delay discounting with the degree of substance use (combined quantity and frequency) and substance-related problems in a sample of (poly-)substance users. Thereby, we aimed to disentangle which of these potential risk factors were associated with the degree of substance use and which ones were specifically relevant for substance-related problems and thus for the diagnosis of SUDs. As expected, sensation seeking was the strongest predictor for the degree of use and the degree of use was the strongest predictor for substance-related problems. Importantly, although the degree of use explains a large part of the variance in substance-related problems, impulsivityrelated traits did explain variance in substance-related problems above and beyond the degree of use.

First, sensation seeking, self-reported urgency and perseverance, as well as delay discounting, explained unique variance in the degree of substance use. Sensation seeking was the strongest predictor, explaining significantly more variance in the degree of substance use than all other predictors. This conforms with the reviewed literature $[8,20,42]$ and indicates that high expressions of both impulsivity and sensation seeking independently, and more so combined, may increase the risk to engage in pronounced substance use. This may already be 
Table 3 Hierarchical Multiple Regression Analyses with the UPPS Subscales and Delay Discounting predicting the Degree of Substance Use and Substance-Related Problems

\begin{tabular}{|c|c|c|c|c|c|c|c|c|c|c|}
\hline \multirow[t]{2}{*}{ Predictors } & \multicolumn{5}{|c|}{ Degree of substance use } & \multicolumn{5}{|c|}{ Substance-related problems } \\
\hline & $\overline{\Delta R^{2}}$ & $F$ & B & SE & $\beta$ & $\overline{\Delta \mathrm{R}^{2}}$ & $\mathrm{~F}$ & B & SE & $\beta$ \\
\hline Step 1 & $.087^{* *}$ & 24.45 & & & & $.017^{*}$ & 4.432 & 4 & & \\
\hline Male gender & & & 11.57 & 2.34 & $.30^{* *}$ & & & 0.75 & 0.35 & $.13^{*}$ \\
\hline Step 2 & & & & & & $.305^{* *}$ & 114.22 & & & \\
\hline Male gender & & & & & & & & -0.23 & 0.31 & -.04 \\
\hline Degree of substance use & & & & & & & & 0.08 & 0.01 & $.58^{* *}$ \\
\hline Step 3 - UPPS & $.144^{* *}$ & 11.70 & & & & $.068^{* *}$ & 6.99 & & & \\
\hline Male gender & & & 7.38 & 2.52 & $.19^{* *}$ & & & -0.06 & 0.33 & -.01 \\
\hline Degree of substance use & & & & & & & & 0.07 & 0.01 & $.49^{* *}$ \\
\hline Urgency & & & 0.52 & 0.21 & $.16^{*}$ & & & 0.11 & 0.03 & $.23^{* *}$ \\
\hline Premeditation & & & -0.09 & 0.28 & -.02 & & & -0.04 & 0.04 & -.06 \\
\hline Perseverance & & & 0.64 & 0.28 & $.15^{*}$ & & & 0.06 & 0.04 & .10 \\
\hline Sensation seeking & & & 0.83 & 0.18 & $.31^{* *}$ & & & 0.03 & 0.02 & .07 \\
\hline Step $4-M C Q$ & $.022^{* *}$ & 7.26 & & & & .001 & 0.30 & & & \\
\hline Male gender & & & 6.40 & 2.51 & $.16^{*}$ & & & -0.08 & 0.34 & -.01 \\
\hline Degree of substance use & & & & & & & & 0.07 & 0.01 & $.48^{* *}$ \\
\hline Urgency & & & 0.43 & 0.21 & $.13^{*}$ & & & 0.11 & 0.03 & $.22^{* *}$ \\
\hline Premeditation & & & -0.08 & 0.27 & -.02 & & & -0.04 & 0.04 & -.06 \\
\hline Perseverance & & & 0.67 & 0.27 & $.15^{*}$ & & & 0.07 & 0.04 & .10 \\
\hline Sensation seeking & & & 0.86 & 0.17 & $.32^{* *}$ & & & 0.03 & 0.02 & .08 \\
\hline $\log (k)$ & & & 3.88 & 1.44 & $.15^{* *}$ & & & 0.10 & 0.19 & .03 \\
\hline Total $R^{2}$ & $.253^{* *}$ & 14.08 & & & & $.391^{* *}$ & 22.83 & & & \\
\hline
\end{tabular}

harmful, e.g. in terms of bodily consequences, but does not necessarily go along with substance-related problems.

Second, the degree of substance use explained about 30\% of the variance in substance-related problems, underlining the undisputed substantial relevance of use patterns for SUDs [43]. Although such a large part of variance was explained by the degree of use, including the UPPS traits into the model added significant explanatory value ( $7 \%$ of total variance in substance-related problems). This was primarily due to the impulsivity-related trait of negative urgency which explained significant unique variance in substancerelated problems above and beyond the degree of substance use, while sensation seeking did not. This finding is in line with prior research [20] but extends the evidence beyond alcohol use and shows that urgency is specifically linked to substance-related problems, the dimension critical for SUDs, even when controlling for substance use and across substances. Building on evidence showing that high levels of urgency precede problematic substance use (e.g. 42), this finding underlines the role of urgency as a potential general risk factor for substance-related problems. Furthermore, this is the first study to combine self-report with behavioral measures of impulsivity using the approach to SUD risk factor research we propose here. This highlights the special role of urgency for SUDs, as this trait was associated with problems even when controlling for the degree of use as well as behavioral impulsivity (delay discounting) and other impulsivity-related traits.

Delay discounting, a behavioral measure of impulsivity, was weakly associated with both the degree of substance use and problems, but the association with problems disappeared when controlling for the degree of use. This is not surprising, considering that delay discounting was only weakly related to substance-related problems in the first place, closely resembling the effect size found in a meta-analysis [27]. This finding indicates that the consistent associations of delay discounting and SUDs described in the literature may be best explained by underlying and confounding differences in the degree of substance use. Although a high degree of use may be inherently harmful, this contradicts the role of delay discounting as a specific risk factor for substance-related problems and thus SUDs discussed in the literature (e.g. $3)$. However, as this is the first study to examine associations of delay discounting with substance-related 
problems controlling for the degree of use, this finding needs replication before drawing final conclusions.

\section{Limitations and future directions}

The dimensional approach we applied in this study proved suitable for identifying differential associations of potential SUD risk factors with substance-related problems controlling for the degree of use. Nonetheless, it requires a longitudinal approach to test whether these factors truly constitute specific risk factors for developing substance-related problems. Furthermore, the delay discounting questionnaire we used in this study uses relatively low monetary rewards. Research suggests that high monetary offers better distinguish substance users from controls [44]. Hence, tasks using higher monetary offers may be more suitable for substance-using populations and delay discounting assessed with such tasks may show a specific association with substance-related problems. Importantly, our sample was predominantly highly educated. It is possible that this acted as a protective factor buffering effects of e.g. delay discounting on substance-related problems. Thus, further studies including more representative samples are warranted to consolidate the generalizability of the associations described in this study. Due to temporal restraints in the online-survey, we did not assess substance-related problems separately for each substance or substance class. Therefore, we could not directly test whether the examined associations differed between substances but rather demonstrated that they were overall significant when all substances were combined. Also, the assessment of substance-related problems was purely based on selfreport, while clinical interviews may provide a more nuanced and comparable picture. For reasons of brevity of the online survey and our decision to combine individual substances to substance classes, we did not assess objective quantities of substance use. The results of this study do not change when using only total frequency rather than the total degree of substance use score as a control variable (see supplementary material for further details). Future studies may include positive urgency, as this facet shows unique associations with substance use outcomes [21] and may thus contribute additional insights.

\section{Conclusions}

This study showed that the impulsivity-related traits of urgency and to some extent perseverance, but not sensation seeking, were specifically linked to substancerelated problems, the dimension that is crucial for SUDs. We applied a dimensional approach to SUD risk factor research examining the associations of potential risk factors with substance-related problems while statistically controlling for the degree of substance use. The present study demonstrated that this approach is suitable for identifying factors specifically linked to substancerelated problems and thus SUDs. Hence, future longitudinal studies using this approach may clarify the role of impulsivity-related traits, alongside others, as risk factors for SUDs. To identify risk factors specifically linked to the development of substance-related problems, rather than predominantly a high degree of use, would convey important implications for practice. A focus on these factors may help to identify individuals within substance using populations who are more in need for preventive measures in order to avoid a transition to problematic substance use. However, longitudinal studies are needed to substantiate the current finding. Furthermore, they may be relevant target points for therapeutic interventions. Taken together, the approach applied in this study may pose a starting point for further research advancing targeted prevention and therapy.

\section{Supplementary Information}

The online version contains supplementary material available at https://doi. org/10.1186/s12888-021-03240-z

Additional file 1: Table S1. Means, Standard Deviations, and Correlations with Confidence Intervals for UPPS subscales, Delay Discounting $(\log (k))$, Substance Use Frequency and Substance-Related Problems. Table S2. Hierarchical Multiple Regression Analyses with the UPPS Subscales and Delay Discounting Predicting Substance Use Frequency and Substance-Related Problems.

\section{Acknowledgements}

Not applicable.

\section{Authors' contributions}

M. H.: Conceptualization, Methodology, Investigation, Formal Analysis, Writing - Original Draft, Project Administration, Funding Acquisition. R. D.: Conceptualization, Methodology, Writing - Review and Editing, Supervision. T. E.: Conceptualization, Resources, Methodology, Writing - Review and Editing, Funding Acquisition, Supervision. All authors contributed to and have approved the final manuscript.

\section{Funding}

This work was supported by a PhD scholarship for Malin Hildebrandt from the Claussen-Simon Stiftung and by the German Research Foundation (Deutsche Forschungsgemeinschaft, grant numbers TRR 265 B01 and EN 906/6). The funding sources had no role in the study design, collection, analysis or interpretation of the data, writing the manuscript, or the decision to submit the paper for publication. Open Access funding enabled and organized by Projekt DEAL.

Availability of data and materials

The dataset supporting the conclusions of this article is available in the OSF repository, DOl https://doi.org/10.17605/OSF.IO/CWNRG, https://mfr.de-1.osf io/render?url=https://osf.io/8wgts/?direct\%26mode=render\%26action= download\%26mode=render.

\section{Declarations}

Ethical approval and consent to participate

The study protocol was approved by the local ethics committee (Ethikkommission an der Technischen Universität Dresden; EK 146042019) and followed the guidelines stated by the Declaration of Helsinki. Participants were thoroughly informed and indicated consent to participate (informed consent) by a button press in the beginning of the online survey. 


\section{Consent for publication}

Not applicable.

\section{Competing interests}

The authors declare that they have no competing interests.

Received: 13 November 2020 Accepted: 26 April 2021

Published online: 07 May 2021

\section{References}

1. Diagnostic and statistical manual of mental disorders. In: American Psychiatric A, American Psychiatric Association DSMTF, editor. DSM-5. Arlington, VA: American Psychiatric Association; 2013.

2. Verdejo-García A, Lawrence AJ, Clark L. Impulsivity as a vulnerability marker for substance-use disorders: review of findings from high-risk research, problem gamblers and genetic association studies. Neurosci Biobehav Rev. 2008:32(4):777-810. https://doi.org/10.1016/j.neubiorev.2007.11.003.

3. Poulton A, Hester R. Transition to substance use disorders: impulsivity for reward and learning from reward. Soc Cogn Affect Neurosci. 2020;15(10): 1182-91. https://doi.org/10.1093/scan/nsz077.

4. Foulds JA, Boden JM, Newton-Howes GM, Mulder RT, Horwood LJ. The role of novelty seeking as a predictor of substance use disorder outcomes in early adulthood. Addiction. 2017;112(9):1629-37. https://doi.org/10.1111/a dd. 13838 .

5. Norbury A, Husain M. Sensation-seeking: dopaminergic modulation and risk for psychopathology. Behav Brain Res. 2015;288:79-93. https://doi.org/10.1 016/j.bbr.2015.04.015.

6. Kozak K, Lucatch AM, Lowe DJE, Balodis IM, MacKillop J, George TP. The neurobiology of impulsivity and substance use disorders: implications for treatment. Ann N Y Acad Sci. 2019;1451(1):71-91. https://doi.org/10.1111/ nyas.13977.

7. Whiteside SP, Lynam DR. The five factor model and impulsivity: using a structural model of personality to understand impulsivity. Personal Individ Differ. 2001;30(4):669-89. https://doi.org/10.1016/S0191-8869(00)00064-7.

8. Miller J, Flory K, Lynam D, Leukefeld C. A test of the four-factor model of impulsivity-related traits. Personal Individ Differ. 2003;34(8):1403-18. https:// doi.org/10.1016/S0191-8869(02)00122-8.

9. Magid V, Maclean MG, Colder CR. Differentiating between sensation seeking and impulsivity through their mediated relations with alcohol use and problems. Addict Behav. 2007;32(10):2046-61. https://doi.org/10.1016/j.a ddbeh.2007.01.015.

10. Whiteside S, Lynam D, Miller J, Reynolds S. Validation of the UPPS impulsive behavior scale: a four-factor model of impulsivity. Eur J Personal. 2005;19(7): 559-74. https://doi.org/10.1002/per.556.

11. Mitchell M, Potenza M. Addictions and personality traits: impulsivity and related constructs. Curr Behav Neurosci Rep. 2014;1(1):1-12. https://doi.org/1 0.1007/s40473-013-0001-y.

12. Duckworth AL, Kern ML. A meta-analysis of the convergent validity of selfcontrol measures. J Res Pers. 2011;45(3):259-68. https://doi.org/10.1016/j. jrp.2011.02.004.

13. Coskunpinar A, Dir AL, Cyders MA. Multidimensionality in impulsivity and alcohol use: a meta-analysis using the UPPS model of impulsivity. Alcohol Clin Exp Res. 2013;37(9):1441-50. https://doi.org/10.1111/acer.12131.

14. Patton JH, Stanford MS, Barratt ES. Factor structure of the barratt impulsiveness scale. J Clin Psychol. 1995;51(6):768-74. https://doi.org/10.1 002/1097-4679(199511)51:6<768::AID-JCLP2270510607>3.0.CO;2-1.

15. McAdams KK, Donnellan MB. Facets of personality and drinking in first-year college students. Personal Individ Differ. 2009;46(2):207-12. https://doi.org/1 0.1016/j.paid.2008.09.028

16. Mason WA, Toumbourou JW, Herrenkohl TI, Hemphill SA, Catalano RF, Patton GC. Early age alcohol use and later alcohol problems in adolescents: individual and peer mediators in a bi-national study. Psychol Addict Behav. 2011;25(4):625-33. https://doi.org/10.1037/a0023320.

17. Castellanos-Ryan N, Parent S, Vitaro F, Tremblay RE, Séguin JR. Puberta development, personality, and substance use: a 10-year longitudinal study from childhood to adolescence. J Abnorm Psychol. 2013;122(3):782-96. https://doi.org/10.1037/a0033133.

18. Fernie G, Peeters M, Gullo MJ, Christiansen P, Cole JC, Sumnall H, et al. Multiple behavioural impulsivity tasks predict prospective alcohol involvement in adolescents. Addiction. 2013;108(11):1916-23. https://doi. org/10.1111/add.12283.
19. Lynam DR, Miller JD. Personality pathways to impulsive behavior and thei relations to deviance: results from three samples. J Quant Criminol. 2004; 20(4):319-41. https://doi.org/10.1007/s10940-004-5867-0.

20. Magid V, Colder CR. The UPPS impulsive behavior scale: factor structure and associations with college drinking. Personal Individ Differ. 2007;43(7):192737. https://doi.org/10.1016/j.paid.2007.06.013.

21. Cyders M, Smith G. Emotion-based dispositions to rash action: positive and negative urgency. Psych Bull. 2008;134(6):807-28. https://doi.org/10.1037/a 0013341.

22. Cyders MA, Coskunpinar A, VanderVeen JD. Urgency: A common transdiagnostic endophenotype for maladaptive risk taking. In: The dark side of personality: Science and practice in social, personality, and clinical psychology. Washington, DC: American Psychological Association; 2016. p. 157-88.

23. Peckham AD, Jordan $H$, Silverman A, Jarvi Steele $S$, Björgvinsson $T$, Beard $C$. From urges to action: negative urgency and nonsuicidal self-injury in an acute Transdiagnostic sample. Arch Suicide Res. 2020;24(3):367-83. https:// doi.org/10.1080/13811118.2019.1625831.

24. Mackillop J, Amlung MT, Few LR, Ray LA, Sweet LH, Munafò MR. Delayed reward discounting and addictive behavior: a meta-analysis. Psychopharmacology. 2011;216(3):305-21. https://doi.org/10.1007/s00213011-2229-0.

25. Wesley MJ, Lohrenz T, Koffarnus MN, McClure SM, De La Garza R 2nd, Salas $\mathrm{R}$, et al. Choosing money over drugs: The neural underpinnings of difficult choice in chronic cocaine users. J Addiction. 2014;2014:189853.

26. Sofis MJ, Budney AJ, Stanger C, Knapp AA, Borodovsky JT. Greater delay discounting and cannabis coping motives are associated with more frequent cannabis use in a large sample of adult cannabis users. Drug Alcohol Depend. 2020;207:107820. https://doi.org/10.1016/j.drugalcdep.201 9.107820

27. Amlung M, Vedelago L, Acker J, Balodis I, Mackillop J. Steep delay discounting and addictive behavior: a meta-analysis of continuous associations. Addiction. 2017;112(1):51-62. https://doi.org/10.1111/add.13 535.

28. Claus ED, Kiehl KA, Hutchison KE. Neural and behavioral mechanisms of impulsive choice in alcohol use disorder. Alcohol Clin Exp Res. 2011;35(7): 1209-19. https://doi.org/10.1111/j.1530-0277.2011.01455.x.

29. Lim AC, Cservenka A, Ray LA. Effects of alcohol dependence severity on neural correlates of delay discounting. Alcohol Alcohol. 2017;52(4):506-15. https://doi.org/10.1093/alcalc/agx015.

30. Steward T, Mestre-Bach G, Fernández-Aranda F, Granero R, Perales JC, Navas $J$ F, et al. Delay discounting and impulsivity traits in young and older gambling disorder patients. Addict Behav. 2017;71:96-103. https://doi.org/1 0.1016/j.addbeh.2017.03.001.

31. Murphy C, Mackillop J. Living in the here and now: interrelationships between impulsivity, mindfulness, and alcohol misuse. Psychopharmacology. 2012;219(2):527-36. https://doi.org/10.1007/s00213011-2573-0

32. Cyders MA, Coskunpinar A. Measurement of constructs using self-report and behavioral lab tasks: is there overlap in nomothetic span and construct representation for impulsivity? Clin Psychol Rev. 2011;31(6):965-82. https:// doi.org/10.1016/j.cpr.2011.06.001.

33. Beesdo-Baum K, Zaudig M, First MB, Wittchen HU. SCID-5-CV: Strukturiertes Klinisches Interview für DSM-5-Störungen - Klinische Version : Deutsche Bearbeitung des Structured Clinical Interview for DSM-5 Disorders - Clinician Version von Michael B. First, Janet B.W. Williams, Rhonda S. Karg, Robert L. Spitzer: Hogrefe; 2019

34. Schmidt R, Gay P, D'Acremont M, Van der Linden M. A german adaptation of the UPPS impulsive behavior scale: psychometric properties and factor structure. Swiss J Psychol. 2008;67(2):107-12. https://doi.org/10.1024/1421-01 85.67.2.107.

35. Kämpfe-Hargrave N, Mitte K. A German validation of the UPPS impulsive behavior scale. Eur J Psychol Assess. 2009;25(4):252-9. https://doi.org/10.102 7/1015-5759.25.4.252.

36. Forstmeier S, Maercker A. Selbstkontrolle im höheren Erwachsenenalter: Eine deutsche Version des Delay Discounting Tests von Kirby. Psychother Psychosom Med Psychol. 2011;61(06):e19-33. https://doi.org/10.1055/s0031-1277169.

37. Ballard K, Knutson B. Dissociable neural representations of future reward magnitude and delay during temporal discounting. Neuroimage. 2009;45(1): 143-50. https://doi.org/10.1016/j.neuroimage.2008.11.004. 
38. Gray JC, Amlung MT, Palmer AA, MacKillop J. Syntax for calculation of discounting indices from the monetary choice questionnaire and probability discounting questionnaire. J Exp Anal Behav. 2016;106(2):156-63. https://doi.org/10.1002/jeab.221.

39. Kirby KN, Marakovic NN. Delay-discounting probabilistic rewards: rates decrease as amounts increase. Psychon Bull Rev. 1996;3(1):100-4. https://doi. org/10.3758/BF03210748.

40. R Development Core team. R: a language and environment for statistical computing. Vienna: R Foundation for Statistical Computing; 2010.

41. Fox J, Weisberg S. An $\{R\}$ Companion to Applied Regression. 3rd ed. Thousand Oaks \{CA\}: Sage; 2019.

42. Cyders MA, Flory K, Rainer S, Smith GT. The role of personality dispositions to risky behavior in predicting first-year college drinking. Addiction. 2009; 104(2):193-202. https://doi.org/10.1111/j.1360-0443.2008.02434.X.

43. Moss HB, Chen CM, Yi H-Y. Measures of substance consumption among substance users, DSM-IV abusers, and those with DSM-IV dependence disorders in a nationally representative sample. J Stud Alcohol Drugs. 2012; 73(5):820-8. https://doi.org/10.15288/jsad.2012.73.820

44. Mellis A, Woodford A, Stein J, Bickel W. A second type of magnitude effect: Reinforcer magnitude differentiates delay discounting between substance users and controls: differences in discounting are magnitude dependent. J Exp Anal Behav. 2017;107(1):151-60. https://doi.org/10.1002/jeab.235.

\section{Publisher's Note}

Springer Nature remains neutral with regard to jurisdictional claims in published maps and institutional affiliations.

Ready to submit your research? Choose BMC and benefit from:

- fast, convenient online submission

- thorough peer review by experienced researchers in your field

- rapid publication on acceptance

- support for research data, including large and complex data types

- gold Open Access which fosters wider collaboration and increased citations

- maximum visibility for your research: over $100 \mathrm{M}$ website views per year

At $\mathrm{BMC}$, research is always in progress.

Learn more biomedcentral.com/submissions 